\title{
Pulmonary Alveolar Proteinosis- A Case Report
}

Renee M. Horner

Orlando Regional Medical Center, rburgess3@student.gsu.edu

Douglas S. Gardenhire

Georgia State University, dgardenhire@gsu.edu

Ralph D. Zimmerman

Georgia State University, chip@gsu.edu

Follow this and additional works at: https://nsuworks.nova.edu/ijahsp

Part of the Analytical, Diagnostic and Therapeutic Techniques and Equipment Commons, Biology Commons, Medical Education Commons, and the Microbiology Commons

\section{Recommended Citation}

Horner RM, Gardenhire DS, Zimmerman RD. Pulmonary Alveolar Proteinosis- A Case Report. The Internet Journal of Allied Health Sciences and Practice. 2018 Jan 01;16(1), Article 3.

This Case Study is brought to you for free and open access by the College of Health Care Sciences at NSUWorks. It has been accepted for inclusion in Internet Journal of Allied Health Sciences and Practice by an authorized editor of NSUWorks. For more information, please contact nsuworks@nova.edu. 


\title{
Pulmonary Alveolar Proteinosis- A Case Report
}

\author{
Abstract \\ ABSTRACT
}

Background: Pulmonary Alveolar Proteinosis (PAP) is a rare pulmonary disorder caused by a congregation of excessive lipoproteinaceous material in the alveolar spaces due to impaired surfactant metabolism. The congregation of the protein in the alveolar space leads to difficulty in breathing, impaired pulmonary immunity, and susceptibility to both opportunistic and acquired pulmonary infections. Although Pulmonary Alveolar Proteinosis is rare, there are potential treatments. Whole-lung lavage is the most widely accepted therapy and course of treatment. An additional form of therapy, GM-CSF stimulating therapy, uses recombinant deoxyribonucleic acid (DNA) technology to increase white cell production.

Purpose: The purpose of this case report is to follow a patient through whole-lung lavage therapy to determine outcome and clinical improvement.

Case Description: This case report follows a 55-year-old female patient diagnosed with secondary, idiopathic Pulmonary Alveolar Proteinosis through eight normal saline whole-lung lavages. The patient's treatment was led by a pulmonologist with previous Pulmonary Alveolar Proteinosis experience.

Outcome: By the end of the first four lavages, the patient showed clinical improvement, but during a twomonth break from therapy, symptoms returned. Following the break, the patient underwent four additional lung lavage sessions and experienced similar relief as in previous courses of treatment.

Discussion: The patient did complete an additional eight lavages to wash out excess lipoproteinaceous material to provide a longer period of symptom relief. The additional form of therapy, GM-CSF, is not a therapy option for this patient as the disease was idiopathic in nature. The last available treatment option for this patient is a lung transplant.

\section{Author Bio(s)}

Renee M. Horner, MS, RRT-NPS, RPFT, is a staff therapist at Orlando Regional Medical Center in Orlando, FL. She is also a licensed respiratory care practitioner in the states of Georgia and Florida.

Douglas S. Gardenhire, EdD, RRT-NPS is Chairman and Associate Professor of Respiratory Therapy in the Lewis College of Nursing and Health Professions at Georgia State University in Atlanta, GA. He is also a licensed respiratory care practitioner in the state of Georgia.

Ralph D. Zimmerman, PhD, RRT-NPS is Director of Clinical Edcuation and Assistant Professor of Respiratory Therapy in the Lewis College of Nursing and Health Professions at Georgia State University in Atlanta, GA. $\mathrm{He}$ is also a licensed respiratory care practitioner in the state of Georgia. 


\title{
TIJAHSP \\ The Internet Joumnal of Allied Health Sciences and Practice \\ Dedicated to allied health professional practice and education
}

Vol. 16 No. 1 ISSN 1540-580X

\section{Pulmonary Alveolar Proteinosis- A Case Report}

\author{
Renee M. Horner, MS, RRT-NPS, RPFT1 \\ Douglas S. Gardenhire, EdD, RRT-NPS² \\ Ralph D. Zimmerman, PhD, RRT-NPS²
}

\author{
1. Orlando Regional Medical Center \\ 2. Georgia State University
}

United States

\begin{abstract}
Background: Pulmonary alveolar proteinosis (PAP) is a rare pulmonary disorder caused by a congregation of excessive lipoproteinaceous material in the alveolar spaces due to impaired surfactant metabolism. The congregation of the protein in the alveolar space leads to difficulty in breathing, impaired pulmonary immunity, and susceptibility to both opportunistic and acquired pulmonary infections. Although pulmonary alveolar proteinosis is rare, there are potential treatments. Whole lung lavage is the most widely accepted therapy and course of treatment. An additional form of therapy, granulocyte macrophage colony stimulating factor (GM-CSF), uses recombinant DNA technology to increase white cell production. Purpose: The purpose of this case report is to follow a patient through whole lung lavage therapy to determine outcome and clinical improvement. Case Description: This case report followed a 55-year-old female patient diagnosed with secondary, idiopathic pulmonary alveolar proteinosis through 8 normal saline whole-lung lavages. The patient's treatment was led by a pulmonologist with previous pulmonary alveolar proteinosis experience. Outcome: By the end of the first 4 lavages, the patient showed clinical improvement, but during a two-month break from therapy, symptoms returned. Following the break, the patient underwent 4 additional lung lavage sessions and experienced similar relief as in previous courses of treatment. Discussion: The patient did complete an additional 8 lavages to wash out excess lipoproteinaceous material to provide a longer period of symptom relief. The additional form of therapy, GM-CSF, was not a therapy option for this patient as the disease was idiopathic in nature. The last available treatment option for this patient is a lung transplant.
\end{abstract}

\section{INTRODUCTION}

This case report followed a 55-year-old female through a total of 8 whole-lung lavages to treat idiopathic pulmonary alveolar proteinosis (PAP). The patient underwent one whole lung lavage a week for a month, followed by a two-month recovery period. During the recovery period, symptoms returned, and the patient underwent the remaining 4 additional whole-lung lavages in hopes of washing out abnormal buildup of excessive lipoproteinaceous material to provide symptomatic relief. Before the case study is presented, a brief history of PAP and available therapies is discussed to provide understanding of this disease and its progression process.

\section{Literature Review}

Research dedicated to pulmonary diseases is expansive, but there is limited peer reviewed, standardized literature available on PAP treatment as this disease is rare. The databases used for this study included EBSCO-host, PUBMED, MEDLINE, and CINAHL. The search was limited by searching for articles that were composed only in English or English and another language with the date range being 1950 through 2017.

PAP was first described by Rosen and colleagues in 1958. ${ }^{1}$ Primary acquired PAP affects approximately 0.37 per 100,000 persons, affecting about 3.7 million individuals globally. ${ }^{2} \mathrm{PAP}$ is a pulmonary disorder caused by impaired surfactant metabolism and homeostasis that causes an excessive accumulation of lipoproteinaceous substance in the alveolar space.3.4 The excess accumulation of lipoproteinaceous material leads to an abnormal buildup in the alveolar space. This abnormal buildup negatively

(C) The Internet Journal of Allied Health Sciences and Practice, 2018 
affects the diffusion capacity of gas exchange and leads to an array of pulmonary symptoms. ${ }^{4}$ Patients can be stable and asymptomatic, but more often, patients experience dyspnea, cough, and difficulty breathing. ${ }^{2}$ Further disease progression may result in respiratory failure. ${ }^{4}$

Surfactant is a substance composed mostly of phospholipids and is produced and stored in the lungs by alveolar type II pneumocytes. ${ }^{1-4}$ When released by the type II pneumocytes, the phospholipids will interact with surfactant proteins and line the alveoli surface to reduce surface tension, which allows for easier distension, prevents collapse, and allows for easier gas exchange. ${ }^{3}$ Approximately $70 \%$ to $80 \%$ of the surfactant phospholipids released are not activated and are recycled by the type II pneumocytes. ${ }^{2,4}$ The surfactant activated by the proteins is later phagocytosed by alveolar macrophages or absorbed by the lymphatic system.

Granulocyte macrophage colony stimulating factor (GM-CSF) is a natural chemical that binds to receptors on macrophages and alveolar type II pneumocytes. Once bound, GM-CSF stimulates the terminal differentiation of the macrophages and their ability to uptake and phagocytize the surfactant. ${ }^{4}$ Patients diagnosed with PAP have developed IgG antibodies that neutralize GM-CSF and cause alveolar macrophages to lose their ability to phagocytize activated surfactant. ${ }^{3}$ This impaired process leads to an accumulation of used activated surfactant in the alveolar space and the manifestation of symptoms.

This deactivation of GM-CSF can be due to a recessive autoimmune disorder or an acquired disease, including dust inhalation or other infections ${ }^{4}$ Common dust inhalants include silica, cotton, cement, titanium, and cellulose. Nitrogen dioxide inhalation can also cause GM-CSF abnormalities. ${ }^{3}$ There are many infectious agents that can be an underlying cause for PAP, but the most common are Nocardia, Cytomegalovirus, Pneumocystis jirovecii, and Mycobacterium tuberculosis. ${ }^{1,3,5}$ Exactly how exposure to irritants, infectious agents, and nitrogen dioxide leads to the development of PAP is not known at this time. As with most pulmonary disorders, PAP is divided into mild or severe categories. Mild cases are asymptomatic with a $\mathrm{PaO} 2$ greater than $65 \mathrm{~mm} \mathrm{Hg}$ while severe cases are symptomatic with a PaO2 less than $65 \mathrm{~mm} \mathrm{Hg}$ and P (A-a)O2 greater than 40 $\mathrm{mm} \mathrm{Hg} .2,3$

Diagnosis begins with a physical exam and chest $\mathrm{X}$-ray. The chest X-ray often shows bilateral perihilar infiltrates. ${ }^{4} \mathrm{~A}$ follow-up computerized topography (CT) scan can be used to confirm the presence of perihilar and diffuse infiltrates, appearing as patchy ground-glass markings with interlobular thickening. An open lung or bronchial biopsy can be performed to further confirm the diagnosis. ${ }^{4}$ While bronchial biopsy can confirm diagnosis, open lung biopsy is considered to be the gold standard. ${ }^{4}$ Once a sample is obtained, a periodic-acid-Shiff-staining (PAS) method is utilized to confirm the diagnosis of PAP. ${ }^{2,6}$ Although Rosen and colleagues stated there was no specific geographic region, race, or gender that are at greater risk of developing PAP, there have been more recent reports of the disease being more common in male patients with a ratio of 2.1:1 male to female. ${ }^{2}$ The typical patient experiences symptoms 7 to 10 months prior to diagnosis, and the median age of diagnosis is 39 years old. $2,5,6$

PAP is difficult to diagnose early as its symptoms are similar to many other more common respiratory diseases. The most common symptom is dyspnea with or without a cough. If a cough is present, the patient may produce white, gummy sputum..$^{1,3}$ The physical examination may show inspiratory crackles, and in chronic situations, the patient may present with clubbing and/or cyanosis. ${ }^{2}$ It has been estimated that $10 \%$ to $30 \%$ of patients originally present as asymptomatic and are later diagnosed with PAP after presenting acutely with rapid deterioration into respiratory failure. ${ }^{4}$ This deterioration is generally due to an opportunistic pulmonary infection, ${ }^{1,2}$ and if an infection is present, the patient commonly presents with a fever. Rare manifestations include hemoptysis, chest pain, pneumothorax, and cor pulmonale. ${ }^{4}$

As with most pulmonary diseases and disorders, treatment and management of the disease process depends on the etiology. Treatment differs for congenital, autoimmune, and acquired forms of PAP as well as for the age of the patient and the severity of the disease. ${ }^{2}$ Mild cases are observed and treated non-aggressively with chest physiotherapy (CPT) and bronchodilators. The two most common treatments for severe cases are whole-lung lavage and/or GM-CSF therapies. ${ }^{4}$ In areas in which advanced medical facilities and treatment such as whole-lung lavage are not available, a bronchial lavage can be performed. ${ }^{5}$ The bronchial lavage includes multiple segmental or lobular lavage procedures performed via a fiberoptic bronchoscope and is considered to be a bridge procedure that is used until a whole-lung lavage can be performed. ${ }^{7}$ If these treatments do not correct the symptoms, a lung transplant may be the next therapeutic option. ${ }^{2}$

Whole-lung lavage consists of a large volume bronchial-alveolar lavage (BAL) in which 1 to 1.5 liters of normal saline are instilled using a double-lumen endotracheal tube (up to a total of 20 liters). ${ }^{8}$ The patient is placed under general anesthesia,

(c) The Internet Journal of Allied Health Sciences and Practice, 2018 
intubated with a double lumen endotracheal tube, then placed onto his/her side with the treatment lung in the superior position. ${ }^{6}$ Once intubation is confirmed, single-lung ventilation is performed, and 1 to 1.5 liters of warmed normal saline are instilled into the non-ventilated lung. ${ }^{8}$ During the procedure, chest physiotherapy is performed manually to the non-ventilated/treatment lung in an effort to loosen the abnormal buildup of proteins in the alveolar space ${ }^{6}$ The patient is then placed in a modified Trendelenburg position, and the fluid is drained from the lung. 7,8 The process is repeated until either 20 liters of saline have been used or the patient no longer tolerates the procedure. Toleration of the procedure is determined by monitoring the patient's vital signs, and if the physician and medical staff determine the vital signs are declining, the procedure is stopped. The fluid drained is initially milky or pink and opaque in appearance and as the instilments continue, the fluid gradually becomes clear. ${ }^{7}$

GM-CSF therapies are used to treat severe autoimmune PAP and involve administering exogenous GM-CSF or suppressing the naturally produced IgG antibody. ${ }^{1-4}$ The response to this therapy is typically slow and the majority of patients show only slight improvement (ie P(A-a) $02>10 \mathrm{~mm} \mathrm{Hg}$ in 4-6 weeks). ${ }^{4}$ The delay in response is attributed to the time required for the immature precursor cells of alveolar macrophages to arrive in the lungs and be differentiate into functional macrophages with properly working GM-CSF. ${ }^{2}$ This therapy is generally more tolerated than whole-lung lavage as the patient does not have to undergo general anesthesia, intubation, mechanical ventilation, or the instillation of such large volumes of saline into the lung. ${ }^{3}$

\section{Background and Purpose}

The patient in this case report is a 55-year-old Caucasian female who was diagnosed with idiopathic PAP during her hospital admission 3 weeks prior to the first whole-lung lavage. This case report presents background on the disease and treatment options to the health care community. Learning about this disease will assist practitioners in identifying possible PAP infections that otherwise would be misdiagnosed. By sharing the X-rays and CT scans of this patient, the health care community is exposed to the clinical process, treatment, and progression of the disease. The description of the treatment process, whole- lung lavage, procedure descriptions, and ventilator settings can be used to explain the technique required to properly perform the therapy.

\section{Case Report}

A 55-year-old Caucasian female with no previous history of pulmonary disorders presented to the clinic with shortness of breath, difficulty in breathing, and a persistent cough for 4 months. The patient denied any recent travel, fever, or environmental allergies. The patient was examined, and diffuse rhonchi and rale breath sounds were auscultated bilaterally. A chest X-ray was performed and showed diffuse infiltrates and mild air bronchograms (see Figure 1). A subsequent CT scan was performed, showing diffuse infiltrates in the alveolar space (see Figure 2). Based on the CT results, a bronchial lung biopsy was scheduled and performed. A sample from the lung parenchyma was obtained and was examined with PAS staining. The PAS stain was positive, and the patient was diagnosed with acquired idiopathic PAP.

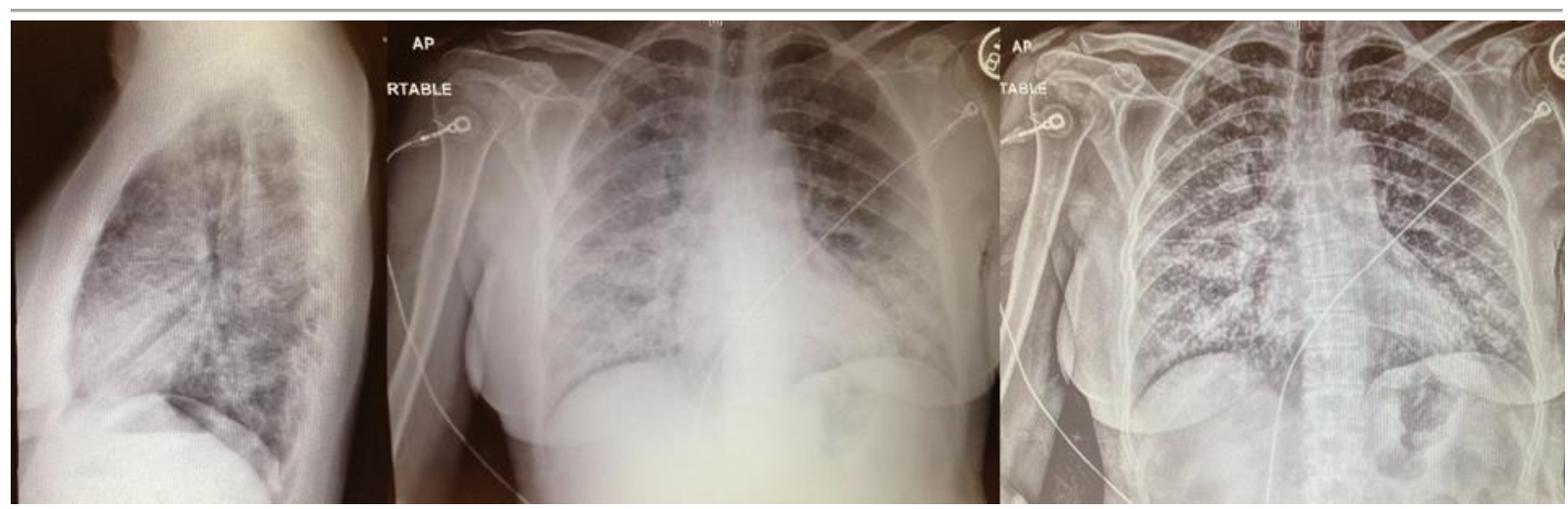

Figure 1. Diagnosis Chest X-Ray Pre-Lavage 


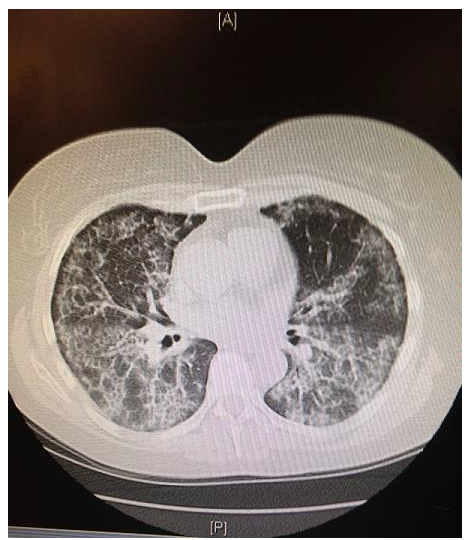

Figure 2. Diagnosis CT Scan Pre-Lavage

\section{Reflections}

Once diagnosed, the patient was scheduled for 4 whole-lung lavage procedures. The patient was sedated with $200 \mathrm{mg}$ propofol and $100 \mathrm{mg}$ of fentanyl for each procedure. The patient was paralyzed with $40 \mathrm{mg}$ of rocuronium before being intubated to prevent unwarranted movement of the pharynx. The patient was then intubated with a 35 French double lumen endotracheal tube and positioned for ventilation, lung lavage, and CPT.

Following confirmation of proper endotracheal tube placement via bronchoscopy, the lung being treated was deflated while the non-treated lung was ventilated in volume control mode. The tidal volume (Vt) was set between $325 \mathrm{~mL}$ to $425 \mathrm{~mL}$, frequency (f) of 14, inspiratory to expiratory ratio (l:E) of 1:2 seconds, a peak end-expiratory pressure (PEEP) of 0 to $4 \mathrm{~cm} \mathrm{H}_{2} \mathrm{O}$, pressure limit of $40 \mathrm{~cm} \mathrm{H}_{2} \mathrm{O}$, and a fractional inspired oxygen percentage (FiO2) of $100 \%$. During the procedure, the patient's ventilator settings and vital signs were monitored and included $\mathrm{Vt}$, $f$, peak inspiratory pressure (PIP), mean airway pressure (MAP1), oxygen saturation (SpO2), end-tidal carbon dioxide (EtCO2), heart rate (HR), temperature (Celsius), blood pressure (BP), and mean arterial pressure (MAP2). With the exception of blood pressure, the patient's ventilator values and vital signs remained stable during each procedure. The average value for each parameter being monitored was: Vt $352 \mathrm{~mL}, \mathrm{f} 12$ breaths/min, PIP $30 \mathrm{~cm} \mathrm{H}_{2} \mathrm{O}, \mathrm{MAP} 112 \mathrm{~cm} \mathrm{H} 2 \mathrm{O}, \mathrm{SpO} 2$ 99\%, EtCO2 $40 \mathrm{~mm} \mathrm{Hg}, \mathrm{HR} 95$ beats/min, temperature 35.5 $\mathrm{C}$, BP $128 / 78 \mathrm{~mm} \mathrm{Hg}$, MAP2 $95 \mathrm{~mm} \mathrm{Hg}$. During 3 out of the 4 treatments, the patient became hypotensive and received $100 \mathrm{mcg}$ of phenylephrine to correct the event.

To ensure proper fluid delivery, the procedure team used a Belmont Rapid Infuser to measure and control the volume of saline at a temperature of $37^{\circ} \mathrm{C}$. Therapeutic volumes of 1 liter to 1.5 liters of normal saline were infused into the deflated lung. Once the therapeutic dose was fully instilled, the patient was placed in Trendelenburg position, and manual CPT was performed across the entire posterior lung field for approximately 3 minutes. The fluid was then allowed drain out via a separate suction catheter into collection containers (see Figure 3).

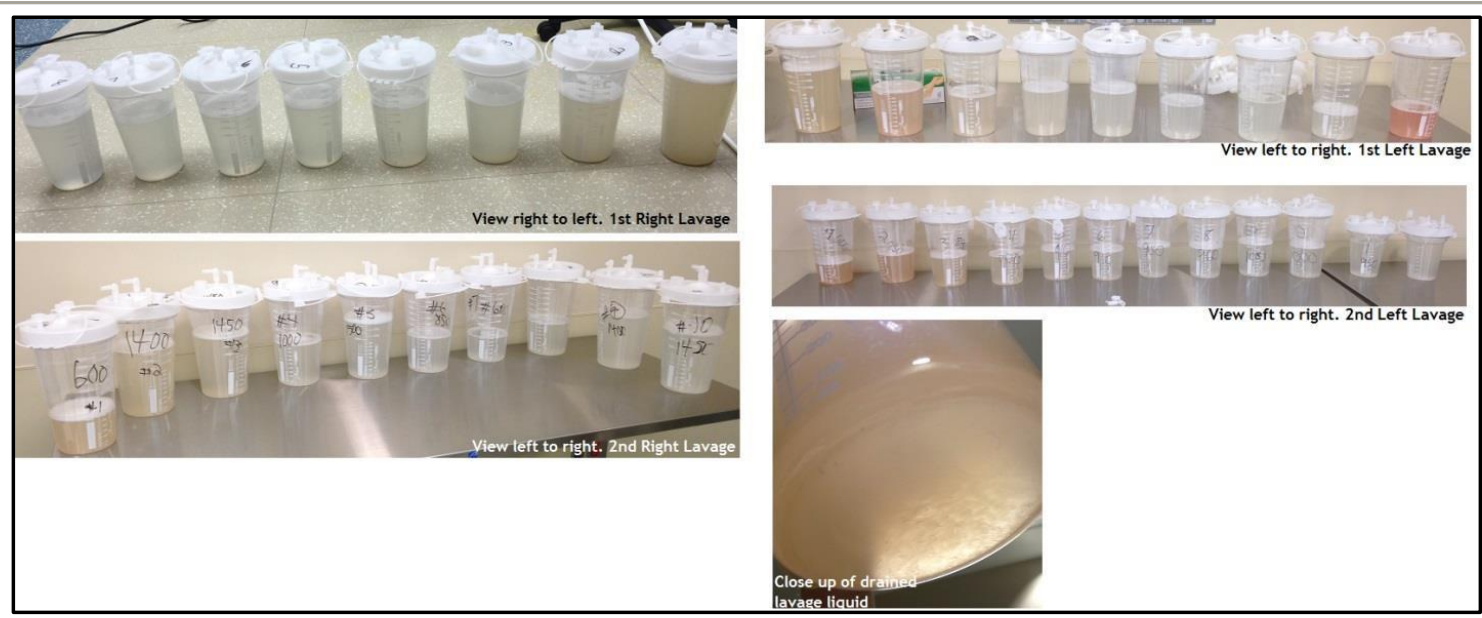

Figure 3. Lavage Washings of Left and Right Lungs 
A Kelly clamp was placed on the drainage suction tubing during the instillation procedure and later moved to the saline delivery tube during drainage. The team monitored the amount of fluid instilled and drained to determine how much fluid was absorbed by the body during the therapy. Once the fluid was fully drained, the patient was placed back in a neutral position, and the process was repeated until the liquid ran clear, or the team decided to end the therapy.

The patient awoke from sedation after the therapy had concluded and was extubated. She was placed on a non- rebreathing oxygen mask at an oxygen flow of 15 liters per minute (LPM) and transported to the intensive care unit (ICU) for overnight evaluation. Post therapy, the patient was placed on 3 LPM to 5 LPM per nasal cannula to keep her oxygen saturation above $92 \%$. She later complained of chest pain on the treated side and difficulty in breathing for around 4 hours. Breath sounds post treatment were improved. The upper lobes were clear and diminished while the lower lobes were still rhonchus with rales but had improved aeration. She was given $12.5 \mathrm{mg}$ promethazine every 6 hours as needed for nausea, hydrocodone every 6 hours as needed for pain, and a $1.25 \mathrm{mg}$ levalbuterol and $0.5 \mathrm{mg}$ ipratropium bromide via small-volume nebulizer every 4 hours for bronchoconstriction. A basic metabolic panel and complete blood count (CBC) were performed after each procedure to verify the patient had no adverse reactions to the therapy. After each procedure, a follow up chest X-ray was performed (see Figure 4). Once all 8 lavages were completed, a follow up CT was performed.

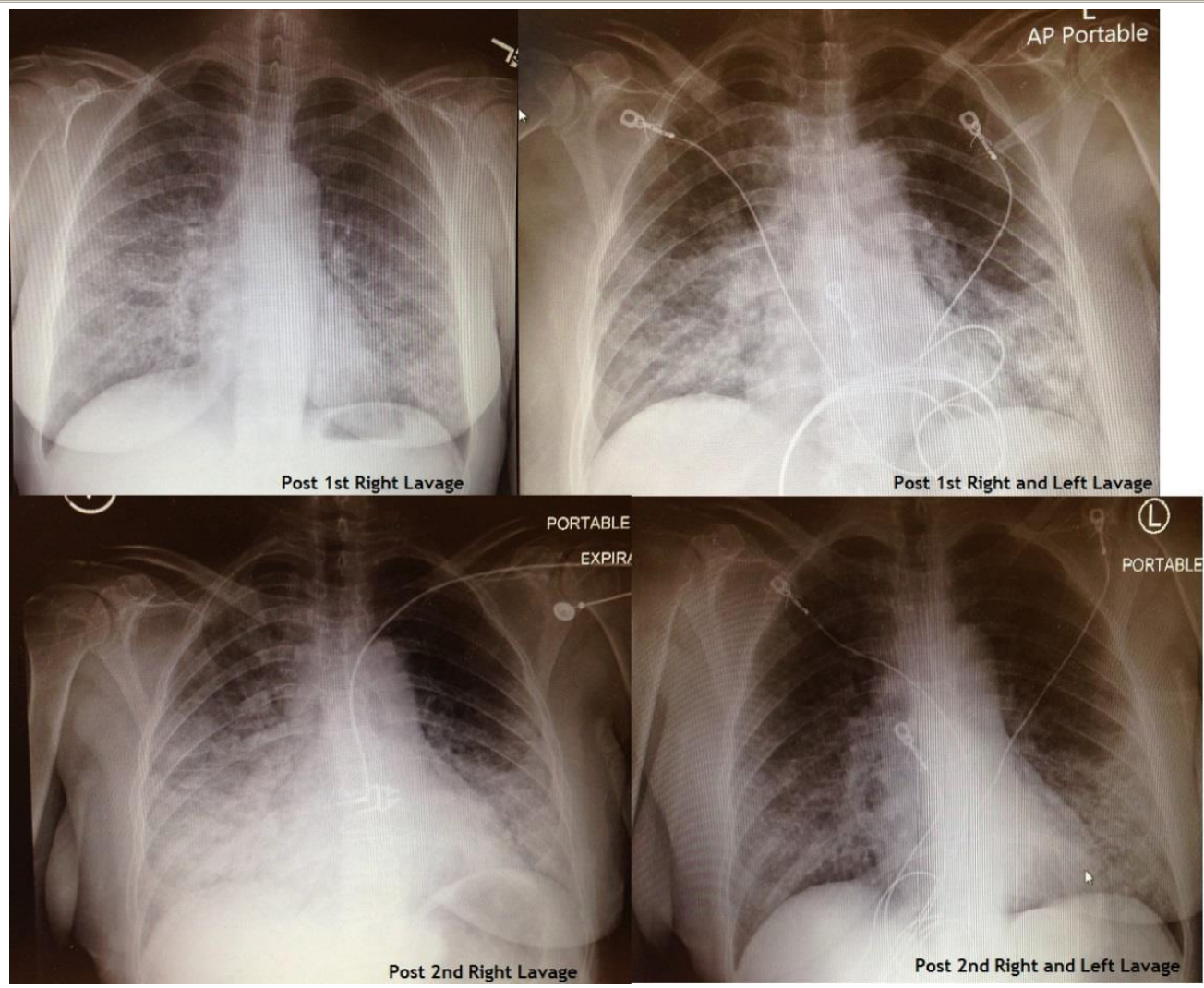

Figure 4. Follow-up Chest X-Rays

The patient was interviewed immediately after the first 4 lavages and stated that she experienced great improvement in both her symptoms and activities of daily life. Prior to the procedures, she required supplemental oxygen around the clock. After the procedures, she only used supplemental oxygen while sleeping. The patient claimed that her work of breathing had greatly improved and that her daily activities were much less tiring.

After a two-month break, the patient was interviewed again and stated that even though her symptoms had returned, they were not at the same level they were prior to receiving treatment. She still required supplemental oxygen at night and occasionally during the day, but not constantly as she did prior to treatment. Her work of breathing also remained improved. She was hopeful the second course of therapy will provide longer relief but knows that a full recovery is not likely. She had hoped for a few more years before needing a lung transplant but has not ruled out a transplant in the future. 


\section{Outcome Analysis}

The patient has successfully completed 8 whole-lung lavages, 4 for each lung. After the first set of 4 lavages $(2$ per lung), the patient was given a two-month break, and during this break, the signs and symptoms returned. The attending physician stated that if the second set of 4 therapeutic lavages did not improve symptoms and slow the progression of the disease, the next step would be to consider a lung transplant. This patient is not eligible for GM-CSF stimulating therapy as she acquired PAP from an unknown source. Therefore, the last form of therapy currently available to treat the disease is a lung transplant. The whole-lung lavage was shown successful in treating the symptoms for a short time, and the patient stated she felt improvement in both work of breathing and lifestyle. The treatment team was hopeful the second therapeutic lavages would be successful in clearing the excess lipoproteinaceousmaterial.

After the first set of 4 lung lavages, the patient had a reduction in her work of breathing, a reduction in her use of oxygen, and reported an improvement in her activities of daily living. During the two-month break, some symptoms returned, and she did not feel as though the therapy had as much of an effect in the long term. The researchers spoke with the pulmonologist prior to the second set of 4 lung lavages, who noted a return of excess lipoproteinaceous material in the lungs. These findings were confirmed by the pulmonologist who noted the accumulation of new lipoproteinaceous material was less severe than prior to the first set of lavages.

\section{Discussion and Conclusion}

The patient outcome correlates with the literature available about the disease and treatment process. Previous research about this disease process and treatment with whole lung lavage showed clinical, functional, and radiological improvements are seen in approximately $80 \%$ of patients after the first treatment.3,5, Other researchers have demonstrated that patients can experience benefits from whole lung lavage for as long as 15 months. However, most patients require repeat lavage with as many as $15 \%$ requiring lavage every 6 months. 3,5 , Even with the need for successive lavages, the procedure is still widely considered to be the gold standard for the treatment of PAP.

In this case report, lung lavage demonstrated the removal of a clinically significant amount of excessive lipoproteinaceous material from the lungs. The post-procedure X-ray showed improved lung aeration and provided clinical documentation for the patient's reduced work of breathing and oxygen use. The researchers concluded that the whole lung lavage was successful in temporarily removing excess lipoproteinaceous material from the patient's lungs.

\section{REFERENCES:}

1. Rosen SH, Castleman B, Liebow AA. Pulmonary alveolar proteinosis. N Engl J Med. 1958;258(23):1123-42. [PMID: 13552931]

2. Sarac S, Milic R, Zolotarevski L, Acimovic S, Tomic I, Plavec G. Primary pulmonary alveolar proteinosis. Vojnosanit Pregl.

3. 2012;69(11):1005-8. doi: 10.2298/VSP1211005S. [PMID: 23311254]

4. Khan A, Agarwal R. Pulmonary alveolar proteinosis. Respir Care. 2011;56(7):1016-28. doi: 10.4187/respcare.01125 [PMID: 21496372]

5. Carden MA, Barman A, Massey G. Pulmonary alveolar proteinosis in association with congenital dyserythropoietic anemia: a case report. Case Rep Pediatr. 2012;2012:624740. doi: 10.1155/2012/624740. [PMID: 22953144]

6. Jin SY, Yun HR, Choi YJ, Park JD, Kim JT,et al. A pediatric case of relapsed pulmonary alveolar proteinosisdespite successful whole lung lavage. Korean J Pediatr. 2017 Jul;60(7):232-6. doi: 10.3345/kjp.2017.60.7.232. [PMID: 28861115]

7. Galhenage J, Weerasinghe B, Dilesha W, Constantine R, Gunasena B. Pulmonary alveolar proteinosis and first successful whole lung lavage in Sri Lanka: a case report. J Med Case Rep. 2017 Mar 8;11(1):62. doi: 10.1186/s13256017-1218-2. [PMID: 28270188

8. Rodrigo D, Rathnapala A, Senaratne W. Therapeutic limited bronchoalveolar lavage with fiberoptic bronchoscopy as a bridging procedure prior to total lung lavage in a patient with pulmonary alveolar proteinosis: a case report. $J$ Med Case Rep. 2015 Apr 29;9:93. doi: 10.1186/s13256-015-0574-z. [PMID: 25925248]

9. Gaëtane M, Chakravarthy R, Armin E. Whole-Lung Lavage for Pulmonary Alveolar Proteinosis. Chest. 2009;136(6):1678-81. doi:10.1378/chest.09-2295. 